\title{
Thromboembolien
}

\section{Bei Tumorpatienten länger mit niedermolekularem Heparin behandeln}

\begin{abstract}
- Bei Tumorpatienten mit venösen
Thromboembolien sind niedermolekulare Heparine (NMH) effektiver als Vitamin-KAntagonisten. Doch die Datenlage dazu ist dünn. Die internationale CATCH-Studie mit Tinzaparin soll nun Gewissheit bringen.

Tiefe Beinvenenthrombosen werden in der Regel akut mit NMH und anschließend je nach Risiko mittel- bis langfristig mit Antikoagulation behandelt. Onkologische Patienten mit idiopathischen Thromboembolien hingegen sollten für drei bis sechs Monate mit niedermolekularem Heparin behandelt werden, so eine S2-Leilinie aus dem Jahr 2010. Im Anschluss ist entweder weiterhin mit Heparin oder mit Vitamin-KAntagonisten zu behandeln, solange die onkologische Erkrankung aktiv ist.

Die Empfehlung, bei onkologischen $\mathrm{Pa}$ tienten mittelfristig mit NMH zu behan-
\end{abstract}

deln, basiert auf drei Studien mit unterschiedlicher Datenqualität. Die beste ist die CLOT-Studie von Lee et al. (N Engl J Med 2003; 349: 146-153). In dieser Studie halbiert eine sechsmonatige Behandlung mit Dalteparin im Vergleich zu einer oralen Antikoagulation das Risiko von Thromboembolien innerhalb von sechs Monaten von $17 \%$ auf $9 \%$, ohne das Blutungsrisiko zu erhöhen. Eine andere Studie mit Tinzaparin (R. Hull et al. Am J. Med 2006; 119: 1062-72) zeigte ebenfalls eine Halbierung des Risikos (von 16\% auf 7\%) innerhalb von zwölf Monaten, jedoch nicht innerhalb von drei Monaten (jeweils 3,3\%).

\section{Große Studie mit Tinzaparin}

Nun hat Leo Pharma mit der CATCH-Studie unter Leitung von Lee et al. eine große internationale Untersuchung mit 900 Tumor-

\section{Ginkgo biloba \\ Gedächtnis-Coach für Gesunde}

- Ginkgo biloba verbessert die Kognition bei Altersdemenz. Aber das Phytotherapeutikum erhöht auch bei Gesunden, die noch nicht das Seniorenalter erreicht haben, die Gedächtnisleistung. Fakten werden exakter erinnert und bleiben länger im Gedächtnis. Dies zeigt eine aktuelle Studie mit 188 gesunden Personen, die entweder den standardisierten Ginkgo-biloba-Extrakt EGb $761^{\oplus}$ (Tebonin $^{\oplus}$ ) in einer Dosierung von $240 \mathrm{mg}$ pro Tag oder Placebo erhielten. Endpunkte waren die Veränderung der Gedächtnisleistung in einem anspruchsvollen standardisierten Free-Recall-Test (freie Erinnerung an eine Liste von Terminen) und in einem weniger anspruchsvollen standardisierten Erinnerungstest (Fahrstrecke).

Im ersten Test erhielten die Probanden eine Liste mit acht Terminen, die sie sich innerhalb von zwei Minuten einprägen sollten, etwa "Dienstag zwischen 11 und 12 Uhr - Thomas in München anrufen". Beim zweiten Test ging es darum, sich anhand eines Films einen Weg einzuprägen und darauf basierend bei einem zweiten Film zu entscheiden, in welcher Richtung man abbiegen muss.

\section{Erinnerungen exakter abrufbar}

Nach sechs Wochen hatten 177 Studienteilnehmer alle Tests vollständig absolviert. In der Verumgruppe wurden mehr Termine korrekt erinnert als in der Placebogruppe. Besonders deutlich war der Ginkgo-Effekt bei der verzögerten Erinnerung. Die Unterschiede zwischen den beiden Gruppen waren statistisch signifikant $(p=0,038$ für die unmittelbare und $p=0,008$ für die verzögerte Erinnerung). In Bezug auf die qualitative Erinnerungsfähigkeit (Fehlerquote) waren die Unterschiede zwischen der Ginkgo- und Placebogruppe noch offensichtlicher $(p=0,092$ für die unmittelbare und $p=0,010$ für die verzögerte Wieder- patienten aufgelegt, die sechs Monate lang entweder mit der vollen Tinzaparindosis oder mit Warfarin behandelt werden. Die Ergebnisse werden 2013 erwartet.

Der Hersteller verspricht sich dadurch zum einen eine klare Evidenz zum Vorgehen bei Tumorpatienten, zum anderen die Zulassung in diesem Kollektiv, wie der Medizinische Direktor Dr. Holger Schilling berichtete. Tinzaparin (innohep ${ }^{\oplus}$ ) ist eine relativ hochmolekulare Substanz unter den $\mathrm{NMH}$ mit einem speziellen enzymatischen Herstellungsprozess. Die Substanz muss in der Thrombosetherapie nur einmal täglich gegeben werden, bei Niereninsuffizienz ist keine Dosisanpassung notwendig.

- Dr. med. Dirk Einecke

Quelle: Fachpressegespräch „LEO Pharma im Portrait: Innovativ von Anfang an“, Neu Isenburg, Mai 2011

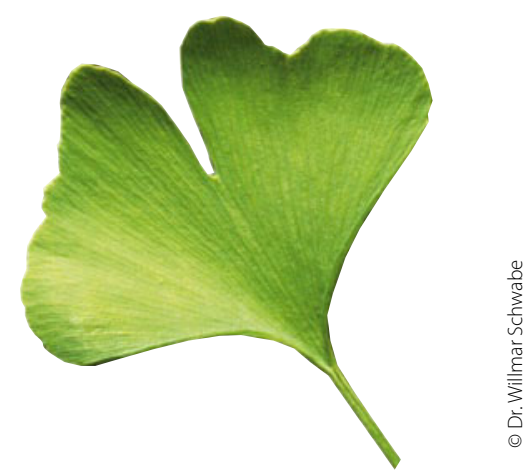

gabe). In dem weniger anspruchsvollen Test zur Fahrstrecken-Erinnerung waren die Ergebnisse dagegen vergleichbar.

Für den Alltag am wichtigsten sind die ausgeprägten Effekte hinsichtlich der korrekten Erinnerung. Denn was nützt es, wenn zwar insgesamt mehr Fakten im Gedächtnis haften bleiben, aber die Erinnerung nicht ganz exakt ist? Um beim Beispiel des Tests zu bleiben: Wer versucht, Thomas am Montag statt am Dienstag anzurufen, wird wahrscheinlich keinen Erfolg haben.

- Dr. med. Kirsten Westphal

Quelle: Kaschel R. Specific memory effects of Ginkgo biloba extract EGb 761 in middle-aged healthy volunteers. Phytomedicine 2011. doi: 10.1016/j.phymed.2011.06.021 